\title{
Monotone and Accretive Vector Fields on Riemannian Manifolds
}

\author{
J.H. Wang, ${ }^{1}$ G. López, ${ }^{2}$ V. Martín-Márquez ${ }^{3}$ and C. $\mathrm{Li}^{4}$
}

Communicated by J.C. Yao

\footnotetext{
${ }^{1}$ Corresponding author, Department of Applied Mathematics, Zhejiang University of Technology, Hangzhou, Zhejiang 310032, P.R. China (wjh@zjut.edu.cn).

${ }^{2}$ Departamento de Análisis Matemático, Universidad de Sevilla, Apdo. 1160, 41080-Sevilla, Spain; partially supported by Ministerio de Ciencia e Innovación, Grant MTM2009-110696-C02-01 and Junta de Andalucía, Grant FQM-127 (glopez@us.es).

${ }^{3}$ Departamento de Análisis Matemático, Universidad de Sevilla, Apdo. 1160, 41080-Sevilla, Spain; partially supported by Ministerio de Ciencia e Innovación, Grants MTM2009-110696-C02-01 and AP2005-1018, and Junta de Andalucía, Grant FQM-127 (victoriam@us.es).

${ }^{4}$ Department of Mathematics, Zhejiang University, Hangzhou 310027, P. R. China; Department of Mathematics, College of Sciences, King Saud University, P. O. Box 2455, Riyadh 11451, Saudi Arabia; partially supported by Ministerio de Ciencia e Innovación, Grant MTM2009-110696-C02-01, Spain; the National Natural Science Foundations of China (Grant No. 10731060) (cli@zju.edu.cn).
} 


\begin{abstract}
The relationship between monotonicity and accretivity on Riemannian manifolds is studied in this paper and both concepts are proved to be equivalent in Hadamard manifolds. As a consequence an iterative method is obtained for approximating singularities of Lipschitz continuous, strongly monotone mappings. We also establish the equivalence between the strong convexity of convex functions and the strong monotonicity of its subdifferentials on Riemannian manifolds. These results are then applied to solve the minimization problem of convex functions on Riemannian manifolds.
\end{abstract}

Keywords: Hadamard manifold, monotone vector field, accretive vector field, singularity, fixed point, iterative algorithm, convex function, minimization problem. 


\section{Introduction}

The concepts of monotonicity and accretivity constitute a valuable tool in studying important operators, such as the gradient or subdifferential of a convex function, which appear in many problems in optimization, equilibrium, variational inequality problems or differential equations; see, for instance, $[1,2,3,4]$ and references therein.

Given a Banach space $E$ with dual space $E^{*}$, recall from [1] that a set-valued operator $A: E \rightarrow 2^{E^{*}}$ is said to be monotone provided that, for any $x, y \in \mathrm{D}(A)$,

$$
\left\langle x^{*}-y^{*}, x-y\right\rangle \geq 0 \text { for any } x^{*} \in A(x) \text { and } y^{*} \in A(y) \text {, }
$$

where $\mathrm{D}(A)$ denotes the domain of $A$ defined by $\mathrm{D}(A):=\{x \in E: A(x) \neq \emptyset\}$. On the other hand, a set-valued operator $A: E \rightarrow 2^{E}$ is said to be accretive if, for each $x, y \in \mathrm{D}(A)$ and $r \geq 0$,

$$
\|x-y\| \leq\|(x+r u)-(y+r v)\| \quad \text { for any } u \in A(x) \text { and } v \in A(y)
$$

One of the most relevant facts in the theory of monotone and accretive operators is that in Hilbert spaces the two classes of operators coincide; see [1].

Some nonconvex constrained minimization problems can be solved by writing them as convex minimization problems in Riemannian manifolds; see, for example, [5, 6, 7, 8, 9]. This fact, together with natural progress in mathematics, have led researchers from different areas, such as optimization, differential equations or fixed point theory, to extend the concepts and techniques which fit in Euclidean spaces to Riemannian manifolds; see,

for example, $[10,11,12]$. These extensions have been developed in the last few years 
often in order to obtain effective algorithms of optimization on Riemannian manifolds; see, for instance, $[6,7,13,14,15]$. In particular, in [7], an algorithm is provided for solving constrained problems in $\mathbb{R}^{n}$ having a constant curvature Hadamard manifold as constraint set. One of the simplest and most powerful among these methods is Newton's. The convergence properties of Newton's method on Riemannian manifolds have been extensively explored in $[16,17,18,19,20,21]$ and the references therein. On the other hand, various derivative-like and subdifferential constructions for nondifferentiable functions on spaces with nonlinear structure have been developed and applied to the study of constrained optimization problems, nonclassical problems of the calculus of variations and optimal control; see $[6,14,15,22]$. They generalized solutions to first-order partial differential equations on Riemannian manifolds and other important classes of spaces without linear structure. Moreover, the extension of the maximal monotonicity to the setting of Riemannian manifolds renders feasible the development of a proximal-type method to approximate singularities of set-valued vector fields on a class of Riemannian manifolds with nonpositive sectional curvatures (i.e., on Hadamard manifolds); see $[6,23,24]$.

The purpose of this paper is to study the relationship among the different concepts of monotone vector fields which previously have been introduced in the framework of Riemannian manifolds, as well as the notion of accretive vector field which is introduced here for the first time in this setting. We also provide an explicit iteration scheme for approximating singularities of strongly monotone vector fields, which is applied for 
solving minimization problems.

The organization of the paper is as follows. In section 2 we introduce basic concepts, results and notations on Riemannian manifolds. In sections 3 and 4, the equivalence of the different definitions of monotonicity on Riemannian manifolds which appear in the literature (e. g., $[5,12,25])$ is proved. As a consequence we show that in the setting of Hadamard manifolds the classes of monotone and strong monotone vector fields coincide, respectively, with the classes of accretive and strongly accretive vector fields introduced in this work. Section 5 is devoted to the study of the existence and approximation of singularities of strongly monotone vector fields. In the last section, section 6 , we introduce the concept of strongly convex functions on Riemannian manifolds and we prove that the subdifferential of this type of functions is strongly monotone. This result is a counterpart of the one proved by Rockafellar in Hilbert spaces [26]. Finally the results of section 5 are applied to obtain the convergence of an iterative method to the minimum of a subprogram to get a minimizer of a convex function [23].

\section{Preliminaries}

In this section we introduce some of the fundamental definitions, properties and notations needed for a comprehensive reading of this paper. This can be found in any text book on Riemannian geometry, for example [13, 27, 28].

Let $M$ be a connected $m$-dimensional manifold and let $x \in M$. The tangent space of $M$ at $x$ is denoted by $T_{x} M$ and the tangent bundle of $M$ by $T M=\bigcup_{x \in M} T_{x} M$, which 
is naturally a manifold. We always assume that $M$ can be endowed with a Riemannian metric $\langle\cdot, \cdot\rangle$, with the corresponding norm denoted by $\|\cdot\|$, to become a Riemannian manifold. Given a piecewise smooth curve $\gamma:[a, b] \rightarrow M$ joining $x$ to $y$ (i.e. $\gamma(a)=x$ and $\gamma(b)=y$, we can define the length of $\gamma$ by $l(\gamma)=\int_{a}^{b}\left\|\gamma^{\prime}(t)\right\| d t$. Then the Riemannian distance $\mathrm{d}(x, y)$, which induces the original topology on $M$, is defined by minimizing this length over the set of all such curves joining $x$ to $y$.

Let $\nabla$ be the Levi-Civita connection associated with $(M,\langle\cdot, \cdot\rangle)$. Let $\gamma$ be a smooth curve in $M$. A vector field $X$ is said to be parallel along $\gamma$ if $\nabla_{\gamma^{\prime}} X=0$. If $\gamma^{\prime}$ itself is parallel along $\gamma$, we say that $\gamma$ is a geodesic, and in this case $\left\|\gamma^{\prime}\right\|$ is constant. When $\left\|\gamma^{\prime}\right\|=1, \gamma$ is said to be normalized. A geodesic joining $x$ to $y$ in $M$ is said to be minimal if its length equals $\mathrm{d}(x, y)$.

A Riemannian manifold is complete if for any $x \in M$ all geodesics emanating from $x$ are defined for all $-\infty<t<\infty$. By the Hopf-Rinow Theorem, we know that if $M$ is complete then any pair of points in $M$ can be joined by a minimal geodesic. Moreover, $(M, d)$ is a complete metric space and bounded closed subsets are compact.

We use $P_{\gamma,, \text {, }}$ to denote the parallel transport on the tangent bundle $T M$ along $\gamma$ with respect to $\nabla$, which is defined by

$$
P_{\gamma, \gamma(b), \gamma(a)}(v)=V(\gamma(b)) \text { for any } a, b \in \mathbb{R} \text { and } v \in T_{\gamma(a)} M,
$$

where $V$ is the unique vector field satisfying $\nabla_{\gamma^{\prime}(t)} V=0$ for all $t$ and $V(\gamma(a))=v$. Then, for any $a, b \in \mathbb{R}, P_{\gamma, \gamma(b), \gamma(a)}$ is an isometry from $T_{\gamma(a)} M$ to $T_{\gamma(b)} M$. We will write $P_{y, x}$ instead of $P_{\gamma, y, x}$ in the case when $\gamma$ is a minimal geodesic joining $x$ to $y$ so no confusion 
arises.

Assuming that $M$ is complete, the exponential map $\exp _{x}: T_{x} M \rightarrow M$ at $x$ is defined by $\exp _{x} v=\gamma_{v}(1, x)$ for each $v \in T_{x} M$, where $\gamma(\cdot)=\gamma_{v}(\cdot, x)$ is the geodesic starting at $x$ with velocity $v$. Then $\exp _{x} t v=\gamma_{v}(t, x)$ for each real number $t$. Note that the mapping $\exp _{x}$ is differentiable on $T_{x} M$ for any $x \in M$.

We will denote by $\mathbf{B}(x, r)$ the open metric ball centered at $x$ with radius $r$. Note that (cf. [27, p.72]) there exist $r>0$ and $\delta>0$ such that, for each $y \in \mathbf{B}(x, r)$, $\exp _{y}(\mathbf{B}(0, \delta)) \supset \mathbf{B}(x, r)$ and $\exp _{y}(\cdot)$ is a diffeomorphism on $\mathbf{B}(0, \delta) \subset T_{y} M$. It is customary to call $\mathbf{B}(x, r)$ a totally normal neighborhood of $x$. The biggest radius $r$ is denoted by $\mathbf{r}_{x}$, that is,

$$
\mathbf{r}_{x}:=\sup \{r>0: \mathbf{B}(x, r) \text { is a totally normal neighborhood of } x\} .
$$

A complete simply-connected Riemannian manifold of nonpositive sectional curvature is called a Hadamard manifold. The following proposition, taken from [28], shows that $\mathbf{r}_{x}=+\infty$ for each $x \in M$ if $M$ is a Hadamard manifold.

Proposition 2.1. Let $M$ be a Hadamard manifold and $x \in M$. Then $\exp _{x}: T_{x} M \rightarrow M$ is a diffeomorphism and, for any two points $x, y \in M$, there exists a unique normalized geodesic joining $x$ to $y$ which is in fact minimal.

Given a nonempty subset $K \subset M$, we denote the closure of $K$ by $\operatorname{cl} K$. The following definition gathers the notions of the different kinds of convexity; items (a) and (b) were defined in [29] whereas items (c) and (d) were, respectively, introduced in [30] and [13]. 
Definition 2.2. A nonempty subset $K \subset M$ is said to be

(a) weakly convex if, for any $x, y \in K$, there exists a minimal geodesic joining $x$ to $y$ contained in $K$;

(b) strongly convex if, for any $x, y \in K$, there is just one minimal geodesic of $M$ joining $x$ to $y$ and it is contained in $K$;

(c) locally convex if, for any $x \in \operatorname{cl} K$, there exists a positive number $\varepsilon>0$ such that $K \cap \mathbf{B}(x, \varepsilon)$ is strongly convex;

(d) totally convex if, for any $x, y \in K$, every geodesic of $M$ joining $x$ to $y$ is contained in $K$.

Clearly, for any nonempty set $K$ in $M$, the following implications hold:

strong convexity (or total convexity) $\Longrightarrow$ weak convexity $\Longrightarrow$ local convexity.

Let $f: M \rightarrow(-\infty,+\infty]$ be a proper extended real-valued function. The effective domain of the function $f$ is denoted by $\mathrm{D}(f)$ and defined by $\mathrm{D}(f):=\{x \in M \mid f(x) \neq$ $+\infty\}$. Given $x, y \in M$, we will denote

$$
\Gamma_{x y}:=\{\gamma:[0,1] \rightarrow M \text { is geodesic such that } \gamma(0)=x \text { and } \gamma(1)=y\}
$$

and

$$
\Gamma_{x y}^{(f)}:=\left\{\gamma \in \Gamma_{x y}: \gamma([0,1]) \subseteq \mathrm{D}(f)\right\}
$$


Definition 2.3. The function $f$ is said to be convex if $\mathrm{D}(f)$ is weakly convex and for any $x, y \in \mathrm{D}(f)$ and $\gamma \in \Gamma_{x y}^{(f)}$ the composition function $f \circ \gamma:[0,1] \rightarrow \mathbb{R}$ is convex; that is,

$$
(f \circ \gamma)(t a+(1-t) b) \leq t(f \circ \gamma)(a)+(1-t)(f \circ \gamma)(b)
$$

for any $a, b \in[0,1]$ and $0 \leq t \leq 1$.

The following proposition, taken from [28], describes the convexity property of the distance function on Hadamard manifolds.

Proposition 2.4. Let $M$ be a Hadamard manifold and $\mathrm{d}: M \times M \rightarrow \mathbb{R}$ the distance function. Then $\mathrm{d}$ is a convex function with respect to the product Riemannian metric; that is, given any pair of geodesics $\gamma_{1}:[0,1] \rightarrow M$ and $\gamma_{2}:[0,1] \rightarrow M$, the following assertion holds for each $t \in[0,1]$ :

$$
\mathrm{d}\left(\gamma_{1}(t), \gamma_{2}(t)\right) \leq(1-t) \mathrm{d}\left(\gamma_{1}(0), \gamma_{2}(0)\right)+t \mathrm{~d}\left(\gamma_{1}(1), \gamma_{2}(1)\right)
$$

In particular, for each $y \in M$, the function $\mathrm{d}(\cdot, y): M \rightarrow \mathbb{R}$ is convex.

\section{Monotone vector fields on Riemannian manifolds}

In the sequel, we always assume that $M$ is a complete connected $m$-dimensional Riemannian manifold. Let $\mathcal{X}(M)$ denote the set of all set-valued vector fields $A: M \rightarrow 2^{T M}$ such that $A(x) \subseteq T_{x} M$, for each $x \in M$, and the domain $\mathrm{D}(A)$ is closed and weakly convex. Given $x, y \in M$, we will denote $\Gamma_{x y}^{(A)}:=\left\{\gamma \in \Gamma_{x y}: \gamma([0,1]) \subseteq \mathrm{D}(A)\right\}$. 
The following definition extends the concepts of monotonicity for operators on Hilbert spaces to set-valued vector fields on Riemannian manifolds, see [24] for the case of singlevalued vector fields and $[5,23]$ for the case of set-valued vector fields.

Definition 3.1. Let $A \in \mathcal{X}(M)$ and $\alpha>0$. The vector field $A$ is said to be

(a) monotone if for any $x, y \in \mathrm{D}(A)$ and $\gamma \in \Gamma_{x y}^{(A)}$, we have

$$
\left\langle u, \gamma^{\prime}(0)\right\rangle \leq\left\langle v, \gamma^{\prime}(1)\right\rangle \text { for any } u \in A(x) \text { and } v \in A(y) \text {; }
$$

(b) $\alpha$-strongly monotone if for any $x, y \in \mathrm{D}(A)$ and $\gamma \in \Gamma_{x y}^{(A)}$, we have

$$
\left\langle u, \gamma^{\prime}(0)\right\rangle-\left\langle v, \gamma^{\prime}(1)\right\rangle \leq-\alpha l^{2}(\gamma) \text { for any } u \in A(x) \text { and } v \in A(y) \text {; }
$$

(c) maximal monotone if it is monotone and the following implication holds for any $x \in M$ and $u \in T_{x} M:$

$$
\left\langle u, \gamma^{\prime}(0)\right\rangle \leq\left\langle v, \gamma^{\prime}(1)\right\rangle, \forall y \in \mathrm{D}(A), \gamma \in \Gamma_{x y}^{(A)} \text { and } v \in A(y) \Longrightarrow u \in A(x)
$$

Remark 3.2. Suppose that $A$ is a monotone vector field and $x \in$ int $\mathrm{D}(A)$. Then, by definition, one has that, for each $v \in T_{x} M$, there exists $\mu>0$ such that $\langle u, v\rangle \leq \mu$ for all $u \in A(x)$. This shows that $A(x)$ is bounded for any $x \in \operatorname{int} \mathrm{D}(A)$.

For our purpose we need to introduce the local version of the previous concepts.

Definition 3.3. Let $A \in \mathcal{X}(M), z_{0} \in \mathrm{D}(A)$ and $\alpha>0$. The vector field $A$ is said to be locally monotone (locally $\alpha$-strongly monotone) at $z_{0}$ if there exists $r>0$ such that, for any $x, y \in \mathbf{B}\left(z_{0}, r\right) \cap \mathrm{D}(A)$, inequality (4) (inequality (5)) holds. 
Remark 3.4. Let $A \in \mathcal{X}(M), z_{0} \in \mathrm{D}(A)$ and $\alpha>0$. By the definition of $\mathbf{r}_{z_{0}}$, one has that $\gamma^{\prime}(0)=\exp _{x}^{-1} y$ and $\gamma^{\prime}(1)=-\exp _{y}^{-1} x$ for any $x, y \in \mathbf{B}\left(z_{0}, \mathbf{r}_{z_{0}}\right) \cap \mathrm{D}(A)$ and $\gamma \in \Gamma_{x y}^{(A)}$. Hence the inequalities in (4) and (5) for the local version can be replaced, respectively, by

$$
\left\langle u, \exp _{x}^{-1} y\right\rangle \leq\left\langle v,-\exp _{y}^{-1} x\right\rangle
$$

and

$$
\left\langle u, \exp _{x}^{-1} y\right\rangle-\left\langle v,-\exp _{y}^{-1} x\right\rangle \leq-\alpha d^{2}(x, y)
$$

Clearly, for $A \in \mathcal{X}(M)$, the global monotonicity implies the corresponding local monotonicity at any point of $\mathrm{D}(A)$. The following proposition shows that the converse is also true.

Theorem 3.5. Let $A \in \mathcal{X}(M)$ and $\alpha>0$. Then $A$ is monotone ( $\alpha$-strongly monotone) if and only if $A$ is locally monotone (locally $\alpha$-strongly monotone) at each point of $\mathrm{D}(A)$. Proof. Only the assertion for the monotonicity case is proved here, since the proof for the strongly monotonicity case is similar.

It is obvious that if $A$ is monotone then it is locally monotone at each point of $\mathrm{D}(A)$. Conversely, assume that $A$ is locally monotone at each point of $\mathrm{D}(A)$. In order to prove that $A$ is monotone, let $x, y \in \mathrm{D}(A)$ and consider $u \in A(x), v \in A(y)$ and $\gamma \in \Gamma_{x y}^{(A)}$. We have to show that

$$
\left\langle P_{x, y} v-u, \gamma^{\prime}(0)\right\rangle \geq 0
$$

Since $A$ is locally monotone at each point of $\mathrm{D}(A)$, it follows that for each $t \in[0,1]$, there exists $r_{t}>0$ such that $\mathbf{B}\left(\gamma(t), r_{t}\right)$ is a totally normal neighborhood of $\gamma(t)$ and for 
any $z_{1}, z_{2} \in \mathbf{B}\left(\gamma(t), r_{t}\right) \cap \mathrm{D}(A)$, the following assertion holds:

$$
\left\langle P_{z_{1}, z_{2}} w_{2}-w_{1}, \exp _{z_{1}}^{-1} z_{2}\right\rangle \geq 0 \quad \text { for each } w_{1} \in A\left(z_{1}\right) \text { and } w_{2} \in A\left(z_{2}\right)
$$

Noting that $\gamma([0,1])$ is a compact subset of $\mathrm{D}(A)$, there exist $0 \leq t_{1}<t_{2}<\cdots<t_{n} \leq 1$ such that the family $\left\{\mathbf{B}\left(\gamma\left(t_{i}\right), r_{t_{i}}\right) \cap \mathrm{D}(A)\right\}$ is a cover of $\gamma([0,1])$; that is,

$$
\gamma([0,1]) \subset \cup_{i=1}^{n}\left(\mathbf{B}\left(\gamma\left(t_{i}\right), r_{i}\right) \cap \mathrm{D}(A)\right)
$$

Without loss of generality, we may assume that $\left\{\mathbf{B}\left(\gamma\left(t_{i}\right), r_{t_{i}}\right) \cap \mathrm{D}(A)\right\}$ is minimal, in other words, any proper sub-family of $\left\{\mathbf{B}\left(\gamma\left(t_{i}\right), r_{t_{i}}\right) \cap \mathrm{D}(A)\right\}$ is not a cover of $\gamma([0,1])$. Thus we can choose $0=s_{0}<s_{1}<s_{2}<\ldots<s_{n-1}<s_{n}=1$ such that

$$
\gamma\left(s_{i}\right) \in \mathbf{B}\left(\gamma\left(t_{i}\right), r_{t_{i}}\right) \cap \mathbf{B}\left(\gamma\left(t_{i+1}\right), r_{t_{i+1}}\right) \cap \mathrm{D}(A) \quad \text { for each } 1 \leq i \leq n-1
$$

Take $v_{i} \in A\left(\gamma\left(s_{i}\right)\right)$ for each $1 \leq i \leq n-1$ and write $v_{0}=u, v_{n}=v$. Note that $\left(s_{i}-s_{i-1}\right) \gamma^{\prime}\left(s_{i-1}\right)=\exp _{\gamma\left(s_{i-1}\right)}^{-1} \gamma\left(s_{i}\right)$ for each $1 \leq i \leq n$. This together with (8) imply that

$$
\left\langle P_{\gamma\left(s_{i-1}\right), \gamma\left(s_{i}\right)} v_{i}-v_{i-1},\left(s_{i}-s_{i-1}\right) \gamma^{\prime}\left(s_{i-1}\right)\right\rangle \geq 0 \quad \text { for each } 1 \leq i \leq n
$$

Consequently,

$$
\left\langle P_{x, y} v-u, \gamma^{\prime}(0)\right\rangle=\sum_{i=1}^{n}\left\langle P_{\gamma\left(s_{i-1}\right), \gamma\left(s_{i}\right)} v_{i}-v_{i-1},\left(s_{i}-s_{i-1}\right) \gamma^{\prime}\left(s_{i-1}\right)\right\rangle \geq 0
$$

and inequality (7) is proved.

The following lemma is an essential tool to study the relationship between monotonicity and accretivity. 
Lemma 3.6. Let $z_{0} \in M$ and let $x, y \in \mathbf{B}\left(z_{0}, \mathbf{r}_{z_{0}}\right)$ with $x \neq y$. Then

$$
\left(\frac{\mathrm{d}}{\mathrm{d} s} \mathrm{~d}\left(\exp _{x} s u, \exp _{y} s v\right)\right)_{s=0}=\frac{1}{\mathrm{~d}(x, y)}\left(-\left\langle u, \exp _{x}^{-1} y\right\rangle+\left\langle v,-\exp _{y}^{-1} x\right\rangle\right)
$$

for any $u \in T_{x} M$ and $v \in T_{y} M$.

Proof. Let $\varepsilon>0$ be such that for each $s \in(-\varepsilon, \varepsilon)$, $\exp _{x} s u, \exp _{y} s v \in \mathbf{B}\left(z_{0}, \mathbf{r}_{z_{0}}\right)$. Let $f:(-\varepsilon, \varepsilon) \times[0,1] \rightarrow M$ be the function defined by

$$
f(s, t)=\exp _{\exp _{x} s u} t\left(\exp _{\exp _{x} s u}^{-1} \exp _{y} s v\right) \quad \text { for each }(s, t) \in(-\varepsilon, \varepsilon) \times[0,1]
$$

Let $\gamma \in \Gamma_{x y}$ be a minimal geodesic. Then $\gamma \subset \mathbf{B}\left(z_{0}, \mathbf{r}_{z_{0}}\right)$ and $\gamma$ can be expressed as

$$
\gamma(t)=\exp _{x} t\left(\exp _{x}^{-1} y\right) \quad \text { for each } t \in[01]
$$

It follows that

$$
\gamma^{\prime}(0)=\exp _{x}^{-1} y \quad \text { and } \quad \gamma^{\prime}(1)=-\exp _{y}^{-1} x
$$

Since the exponential map exp is differentiable and $f(0, \cdot)=\gamma(\cdot), f$ is a variation of $\gamma$ and $V(\cdot)=\frac{\partial f}{\partial s}(0, \cdot)$ is the variational field of $f$. In particular,

$$
V(0)=\frac{\partial f}{\partial s}(0,0)=u \quad \text { and } \quad V(1)=\frac{\partial f}{\partial s}(0,1)=v
$$

Note that for each $s \in(-\varepsilon, \varepsilon)$, the parameterized curve $f_{s}:[0,1] \rightarrow M$ given by $f_{s}(\cdot)=f(s, \cdot)$ is a geodesic and so $\left\|\frac{\partial f}{\partial t}(s, \cdot)\right\|$ is a constant. Therefore

$$
\left\|\frac{\partial f}{\partial t}(s, \cdot)\right\|=\left\|\exp _{\exp _{x} s u}^{-1} \exp _{y} s v\right\|=d\left(\exp _{x} s u, \exp _{y} s v\right) \quad \text { for each } s \in(-\varepsilon, \varepsilon) .
$$

Define $L:(-\varepsilon, \varepsilon) \rightarrow \mathbb{R}$ by

$$
L(s)=\int_{0}^{1}\left\|\frac{\partial f}{\partial t}(s, t)\right\| \mathrm{d} t \quad \text { for each } s \in(-\varepsilon, \varepsilon) .
$$


Then, by the first variational formula, see e.g. [28, p.38, Proposition 2.5],

$$
\begin{aligned}
\left(\frac{\mathrm{d}}{\mathrm{d} s} L(s)\right)_{s=0} & =\frac{1}{l(\gamma)}\left(-\int_{0}^{1}\left\langle V(t), \frac{\mathrm{D}}{\mathrm{d} t} \frac{\mathrm{d} \gamma}{\mathrm{d} t}\right\rangle \mathrm{d} t-\left\langle V(0), \frac{\mathrm{d} \gamma}{\mathrm{d} t}(0)\right\rangle+\left\langle V(1), \frac{\mathrm{d} \gamma}{\mathrm{d} t}(1)\right\rangle\right) \\
& =\frac{1}{d(x, y)}\left(-\left\langle\frac{\partial f}{\partial s}(0,0), \gamma^{\prime}(0)\right\rangle+\left\langle\frac{\partial f}{\partial s}(0,1), \gamma^{\prime}(1)\right\rangle\right),
\end{aligned}
$$

where the second equality holds because $\gamma$ is a geodesic and $\frac{\mathrm{D}}{\mathrm{d} t} \frac{\mathrm{d} \gamma}{\mathrm{d} t}=0$. Then, bearing in mind that

$$
\left(\frac{\mathrm{d}}{\mathrm{d} s} \mathrm{~d}\left(\exp _{x} s u, \exp _{y} s v\right)\right)_{s=0}=\left(\frac{\mathrm{d}}{\mathrm{d} s} L(s)\right)_{s=0},
$$

equality (10) follows from (11), (12) and (15).

The following characterization of the local monotonicity is a direct consequence of Definition 3.3 and Lemma 3.6.

Theorem 3.7. Let $A \in \mathcal{X}(M), z_{0} \in M$ and $\alpha>0$. Then the following assertions hold.

(i) A is locally monotone at $z_{0}$ if and only if there exists $r>0$ such that, for any

$$
\begin{aligned}
& x, y \in \mathbf{B}\left(z_{0}, r\right) \cap \mathrm{D}(A), \\
& \quad\left(\frac{\mathrm{d}}{\mathrm{d} s} \mathrm{~d}\left(\exp _{x}(s u), \exp _{y}(s v)\right)\right)_{s=0} \geq 0 \quad \text { for any } u \in A(x) \text { and } v \in A(y) .
\end{aligned}
$$

(ii) A is locally $\alpha$-strongly monotone at $z_{0}$ if and only if there exists $r>0$ such that, for any $x, y \in \mathbf{B}\left(z_{0}, r\right) \cap \mathrm{D}(A)$,

$$
\left(\frac{\mathrm{d}}{\mathrm{d} s} \mathrm{~d}\left(\exp _{x}(s u), \exp _{y}(s v)\right)\right)_{s=0} \geq \alpha \mathrm{d}(x, y) \quad \text { for any } u \in A(x) \text { and } v \in A(y) \text {. }
$$

In the particular case when $M$ is a Hadamard manifold, as noted earlier, $\mathbf{r}_{z_{0}}=+\infty$ for each $z_{0} \in M$. Thus, by Lemma 3.6, we have the following corollary. 
Corollary 3.8. Let $A \in \mathcal{X}(M)$ and $\alpha>0$. Suppose that $M$ is a Hadamard manifold. Then $A$ is monotone ( $\alpha$-strongly monotone) if and only if, for any $x, y \in \mathrm{D}(A)$, inequality (16) (inequality (17)) holds.

Remark 3.9. It is worth mentioning that in [12] a vector field $A \in \mathcal{X}(M)$ is said to be monotone if inequality (16) holds for any $x, y \in \mathrm{D}(A)$. In the case when $M$ is a Hadamard manifold, we see from Corollary 3.8 that this alternative definition coincides with the one considered in this paper.

\section{Accretive vector fields on Riemannian manifolds}

We begin with the following definition which extends the concepts of accretivity to set-valued vector fields on Riemannian manifolds.

Definition 4.1. Let $A \in \mathcal{X}(M)$ and $\alpha>0$. The vector field $A$ is said to be

(a) accretive if for any $x, y \in \mathrm{D}(A)$ and each $r \geq 0$ we have that

$$
\mathrm{d}(x, y) \leq \mathrm{d}\left(\exp _{x}(r u), \exp _{y}(r v)\right) \quad \text { for any } u \in A(x) \text { and } v \in A(y) \text {; }
$$

(b) $\alpha$-strongly accretive if for any $x, y \in \mathrm{D}(A)$ and each $r \geq 0$ we have that

$$
(1+\alpha r) \mathrm{d}(x, y) \leq \mathrm{d}\left(\exp _{x}(r u), \exp _{y}(r v)\right) \quad \text { for any } u \in A(x) \text { and } v \in A(y)
$$

(c) m-accretive if it is accretive and

$$
\bigcup_{x \in \mathcal{D}(A)}\left(\bigcup_{u \in A(x)} \exp _{x} u\right)=M .
$$


Definition 4.2. Let $A \in \mathcal{X}(M), z_{0} \in \mathrm{D}(A)$ and $\alpha>0$. The vector field $A$ is said to be locally accretive (locally $\alpha$-strongly accretive) at $z_{0}$ if there exists $r_{1}, r_{2}>0$ such that, for any $x, y \in \mathbf{B}\left(z_{0}, r_{1}\right) \cap \mathrm{D}(A)$ and each $0 \leq r \leq r_{2}$, inequality (18) (inequality (19)) holds.

The following theorem describes the relationships between the notions of accretivity and the monotonicity.

Theorem 4.3. Let $A \in \mathcal{X}(M), z_{0} \in \mathrm{D}(A)$ and $\alpha>0$. Then the following assertions hold.

(i) If $A$ is locally accretive at $z_{0}$ then $A$ is locally monotone at $z_{0}$.

(ii) If $A$ is locally $\alpha$-strongly accretive at $z_{0}$ then $A$ is locally $\alpha$-strongly monotone at $z_{0}$. Conversely, if $A$ is locally $\alpha$-strongly monotone at $z_{0}$ then $A$ is locally $\alpha^{\prime}$-strongly accretive at $z_{0}$ for each $0<\alpha^{\prime}<\alpha$.

Proof. (i). Assume that $A$ is locally accretive at $z_{0}$. Then there exists $r_{1}, r_{2}>0$ such that for any $x, y \in \mathbf{B}\left(z_{0}, r_{1}\right) \cap \mathrm{D}(A)$ and each $u \in A(x)$ and $v \in A(y)$, we have that

$$
\mathrm{d}(x, y) \leq \mathrm{d}\left(\exp _{x}(r u), \exp _{y}(r v)\right) \quad \text { for each } r_{2} \geq r \geq 0
$$

which means that

$$
\left(\frac{\mathrm{d}}{\mathrm{d} s} \mathrm{~d}\left(\exp _{x} s u, \exp _{y} s v\right)\right)_{s=0} \geq 0 .
$$

This together with Theorem 3.7 implies that $A$ is locally monotone.

(ii). Assume that $A$ is localy $\alpha$-strongly accretive at $z_{0}$. Then the same argument we did for the proof of (i) shows that $A$ is locally $\alpha$-strongly monotone at $z_{0}$. 
Conversely, assume that $A$ is local $\alpha$-strongly monotone at $z_{0}$ and let $0<\alpha^{\prime}<\alpha$. Then, there exists $r_{1}>0$ such that for any $x, y \in \mathbf{B}\left(z_{0}, r_{1}\right) \cap \mathrm{D}(A)$, and any $u \in A(x), v \in$ $A(y)$, one has

$$
-\left\langle u, \exp _{x}^{-1} y\right\rangle+\left\langle v,-\exp _{y}^{-1} x\right\rangle \geq \alpha \mathrm{d}^{2}(x, y) .
$$

Let $x, y \in \mathbf{B}\left(z_{0}, r_{1}\right) \cap \mathrm{D}(A)$. Without loss of generality, assume that $x \neq y$ and so $\mathrm{d}(x, y)>0$. Let $u \in A(x)$ and $v \in A(y)$ be arbitrary. Then by Lemma 3.6 we get that

$$
\left(\frac{\mathrm{d}}{\mathrm{d} s} \mathrm{~d}\left(\exp _{x} s u, \exp _{y} s v\right)\right)_{s=0}=\frac{1}{\mathrm{~d}(x, y)}\left(-\left\langle u, \exp _{x}^{-1} y\right\rangle+\left\langle v,-\exp _{y}^{-1} x\right\rangle\right) \geq \alpha \mathrm{d}(x, y)
$$

This means that there exists $r_{2}>0$ such that

$$
\mathrm{d}\left(\exp _{x} r u, \exp _{y} r v\right)-\mathrm{d}(x, y)>r \alpha^{\prime} \mathrm{d}(x, y) \quad \text { for each } 0<r \leq r_{2} ;
$$

hence

$$
\left(1+\alpha^{\prime} r\right) \mathrm{d}(x, y) \leq \mathrm{d}\left(\exp _{x} r u, \exp _{y} r v\right) \quad \text { for each } 0 \leq r \leq r_{2} .
$$

Therefore, $A$ is local $\alpha^{\prime}$-strongly accretive at $z_{0}$ and the proof is complete.

Combining Theorems 3.5 and 4.3, we have the following corollary.

Corollary 4.4. Let $A \in \mathcal{X}(M)$ and $\alpha>0$. If $A$ is accretive ( $\alpha$-strongly accretive), then $A$ is monotone ( $\alpha$-strongly monotone).

In the particular case when $M$ is a Hadamard manifold, the notions of accretivity and monotonicity can be proved to be equivalent, as we show in Theorem 4.6 below. 
Lemma 4.5. Let $M$ be a Hadamard manifold. Let $x, y \in M$ with $x \neq y$ and let $u \in T_{x} M, v \in T_{y} M$. Then the following inequality holds for each $r>0$ :

$$
\mathrm{d}\left(\exp _{x}(r u), \exp _{y}(r v)\right) \geq \mathrm{d}(x, y)+r\left(\frac{\mathrm{d}}{\mathrm{d} s} \mathrm{~d}\left(\exp _{x}(s u), \exp _{y}(s v)\right)\right)_{s=0} .
$$

Proof. Define the function $g:[0,+\infty) \rightarrow[0,+\infty)$ by

$$
g(s):=\mathrm{d}\left(\exp _{x} s u, \exp _{y} s v\right) \quad \text { for each } s \in[0,+\infty)
$$

Then $g(\cdot)$ is convex by Proposition 2.4 (as $M$ is a Hadamard manifold). Let $r>0$. Then

$$
\frac{g(r)-g(0)}{r} \geq \inf _{r \geq 0} \frac{g(r)-g(0)}{r}=\left(\frac{\mathrm{d}}{\mathrm{d} s} \mathrm{~d}\left(\exp _{x}(s u), \exp _{y}(s v)\right)\right)_{s=0} .
$$

This shows (23) and completes the proof.

Theorem 4.6. Let $M$ be a Hadamard manifold, $A \in \mathcal{X}(M)$ and $\alpha>0$. Then the following assertions hold.

(i) $A$ is accretive ( $\alpha$-strongly accretive) if and only if $A$ is monotone ( $\alpha$-strongly monotone).

(ii) If $A$ is $m$-accretive, then $A$ is maximal monotone. The converse is true provided that $\mathrm{D}(A)=M$.

Proof. (i). Since the proof for the case of $\alpha$-strong accretivity is similar, we only keep the proof here for the case of accretivity. Furthermore, by Corollary 4.4, it is sufficient to prove the sufficient part. To this end, we assume that $A$ is monotone. Let $x, y \in \mathrm{D}(A)$ and $u \in A(x), v \in A(y)$. Then, by Corollary 3.8,

$$
\left(\frac{\mathrm{d}}{\mathrm{d} s} \mathrm{~d}\left(\exp _{x}(s u), \exp _{y}(s v)\right)\right)_{s=0} \geq 0
$$


This together with Lemma 4.5 shows that $A$ is accretive.

(ii). Assume that $A$ is $m$-accretive. In particular, $A$ is accretive and so $A$ is monotone by (i). In order to prove the maximality, we take $x \in M$ and $u \in T_{x} M$, and assume that

$$
\left\langle u, \exp _{x}^{-1} y\right\rangle \leq-\left\langle v, \exp _{y}^{-1} x\right\rangle \quad \text { for any } y \in \mathrm{D}(A) \text { and } v \in A(y) \text {. }
$$

We have to verify that $x \in \mathrm{D}(A)$ and $u \in A(x)$. Suppose on the contrary that it is not the case. Then $x \neq y$ for each $y \in \mathrm{D}(A)$. Thus Lemma 3.6 is applicable to getting that

$$
\left(\frac{\mathrm{d}}{\mathrm{d} s} \mathrm{~d}\left(\exp _{x}(s u), \exp _{y}(s v)\right)\right)_{s=0} \geq 0 \quad \text { for any } y \in \mathrm{D}(A) \text { and } v \in A(y) \text {. }
$$

This together with Lemma 4.5 implies that

$$
\mathrm{d}(x, y) \leq \mathrm{d}\left(\exp _{x} u, \exp _{y} v\right) \quad \text { for any } y \in \mathrm{D}(A) \text { and } v \in A(y) .
$$

On the other hand, since $A$ is $m$-accretive, by (20), there exist $y \in \mathrm{D}(A)$ and $v \in A(y)$ such that $\exp _{x} u=\exp _{y} v$. This together with (27) yields that $x=y$, which is a contradiction.

Conversely, assume that $A$ is maximal monotone and $\mathrm{D}(A)=M$. Then $A$ is monotone, and so accretive by (i). In order to proved that $A$ is $m$-accretive we need to show that (20) is true. To this end,let $y \in M$ and define the set-valued vector field $B: M \rightarrow 2^{T M}$ by

$$
B(x):=A(x)-\exp _{x}^{-1} y \quad \text { for each } x \in M .
$$

It turns out that $B$ is maximal 1-strongly monotone (cf. Example 6.1 of section 6 ) and $\mathrm{D}(B)=M$. Therefore, by [23, Theorem 4.3], there exists a unique singularity $x_{0}$ of $B$, 
that is, $0 \in A\left(x_{0}\right)-\exp _{x_{0}}^{-1} y$. This means that $y \in \cup_{u \in A\left(x_{0}\right)} \exp _{x_{0}} u$. Hence (20) is seen to hold as $y \in M$ is arbitrary.

Remark 4.7. By definition, it is straightforward to see that the accretivity (strongly accretivity) implies the corresponding local accretivity (strongly accretivity) at each point of $\mathrm{D}(A)$. Combining Theorems 3.5 and 4.6, one can deduce that the converse is true in the case when $M$ is a Hadamard manifold. However, we do not know if this is also true in general Riemannian manifolds.

\section{$5 \quad$ Singularities of $\alpha$-strongly monotone vector fields}

In the setting of Banach spaces, iterative methods to approximate singularities of strongly monotone vector fields or, equivalently, fixed points of $\alpha$-strongly pseudo-contractive mappings have been studied by many authors; see, for instance, [31, 32, 33]. The aim of this section is to define and study the convergence of an iterative scheme which is an extension to Riemannian manifolds of the one studied by Chidume (cf. [31]) in Banach spaces.

For the main theorem of this section, we need to extend the notion of the $L$-Lipschitz continuity to the setting of general Riemannian manifolds. Given $L>0$, a single-valued vector field $A \in \mathcal{X}(M)$ is said to be L-Lipschitz continuous if

$$
\left\|P_{\gamma, x, y} A(x)-A(y)\right\| \leq L l(\gamma) \quad \text { for any } x, y \in \mathrm{D}(A) \text { and any } \gamma_{x y} \in \Gamma_{x y}^{(A)}
$$

Theorem 5.1. Let $A \in \mathcal{X}(M)$ be a single-valued, L-Lipschitz continuous and $\alpha$-strongly 
monotone vector field with $\mathrm{D}(A)=M$. Given $x_{0} \in M$, let $\left\{x_{n}\right\}$ be the sequence defined by the algorithm

$$
x_{n+1}=\exp _{x_{n}}\left(-r A\left(x_{n}\right)\right)
$$

where $0<r<\frac{2 \alpha}{L^{2}}$. Then $\left\{x_{n}\right\}$ converges to the unique singularity of $A$.

Proof. Note that the uniqueness of singularity follows from the strong monotonicity of $A$. Below we prove the convergence of the sequence $\left\{x_{n}\right\}$ generated by (29). To do this, let $n \in \mathbb{N}$ and let $\gamma_{n} \in \Gamma_{x_{n}, x_{n+1}}$ be defined by

$$
\gamma_{n}(t):=\exp _{x_{n}}\left(t\left(-r A\left(x_{n}\right)\right)\right) \quad \text { for each } t \in[0,1]
$$

Write $P_{x_{n}, x_{n+1}}=P_{\gamma_{n}, x_{n}, x_{n+1}}$ for simplicity. Since $A$ is $\alpha$-strongly monotone, it follows that

$$
\left\langle P_{x_{n}, x_{n+1}} A\left(x_{n+1}\right)-A\left(x_{n}\right), \gamma_{n}^{\prime}(0)\right\rangle \geq \alpha l^{2}\left(\gamma_{n}\right)
$$

Since $\gamma_{n}^{\prime}(0)=-r A\left(x_{n}\right)$ and $l\left(\gamma_{n}\right)=r\left\|A\left(x_{n}\right)\right\|$, the inequality (30) implies that

$$
\left\langle P_{x_{n}, x_{n+1}} A\left(x_{n+1}\right)-A\left(x_{n}\right), A\left(x_{n}\right)\right\rangle \leq-\alpha r\left\|A\left(x_{n}\right)\right\|^{2}
$$

On the other hand, we have

$$
\begin{aligned}
\left\|A\left(x_{n+1}\right)\right\|^{2}= & \left\|P_{x_{n}, x_{n+1}} A\left(x_{n+1}\right)-A\left(x_{n}\right)+A\left(x_{n}\right)\right\|^{2} \\
= & \left\|P_{x_{n}, x_{n+1}} A\left(x_{n+1}\right)-A\left(x_{n}\right)\right\|^{2}+\left\|A\left(x_{n}\right)\right\|^{2}+ \\
& +2\left\langle P_{x_{n}, x_{n+1}} A\left(x_{n+1}\right)-A\left(x_{n}\right), A\left(x_{n}\right)\right\rangle .
\end{aligned}
$$

Then, from inequality (31) we obtain that

$$
\left\|A\left(x_{n+1}\right)\right\|^{2} \leq\left\|P_{x_{n}, x_{n+1}} A\left(x_{n+1}\right)-A\left(x_{n}\right)\right\|^{2}+\left\|A\left(x_{n}\right)\right\|^{2}-2 \alpha r\left\|A\left(x_{n}\right)\right\|^{2} .
$$


Since $A$ is $L$-Lipschitz continuous, one has

$$
\left\|P_{x_{n}, x_{n+1}} A\left(x_{n+1}\right)-A\left(x_{n}\right)\right\| \leq L l\left(\gamma_{n}\right)=L r\left\|A\left(x_{n}\right)\right\| .
$$

Combining this with the inequality (33), we get that

$$
\left\|A\left(x_{n+1}\right)\right\| \leq\left(1+r\left(L^{2} r-2 \alpha\right)\right)^{\frac{1}{2}}\left\|A\left(x_{n}\right)\right\|=q\left\|A\left(x_{n}\right)\right\|,
$$

where, thanks to the fact that $0<r<\frac{2 \alpha}{L^{2}}$,

$$
q:=\left(1+r\left(L^{2} r-2 \alpha\right)\right)^{\frac{1}{2}}<1 .
$$

Hence, $\lim _{n} A\left(x_{n}\right)=0$. On the other hand, by (29), we have that

$$
\mathrm{d}\left(x_{n+1}, x_{n}\right) \leq r\left\|A\left(x_{n}\right)\right\| \leq r q^{n}\left\|A\left(x_{0}\right)\right\| .
$$

This means that the sequence $\left\{x_{n}\right\}$ converges to some point $x^{*}$ satisfying $A\left(x^{*}\right)=0$ because $\lim _{n} A\left(x_{n}\right)=0$ and $A$ is Lipschitz continuous.

\section{Application to minimization}

In the linear case, an important class of convex functions is the one of the strongly convex functions introduced by Polyak, in [34]. Consider the special case of a Hilbert space $H$ and let $\alpha>0$. Recall that a function $f: H \rightarrow(-\infty,+\infty]$ is said to be $\alpha$-strongly convex if

$$
f((1-t) x+t y) \leq(1-t) f(x)+t f(y)-\frac{1}{2} \alpha t(1-t)\|x-y\|^{2}
$$


for any $x, y \in H$ and $0<t<1$. Rockafellar, in [26], proved that a function $f: H \rightarrow$ $(-\infty,+\infty]$ is $\alpha$-strongly convex if and only if its subdiferential $\partial f$ is $\alpha$-strongly monotone. This section is devoted to an extension of the notion of $\alpha$-strongly convexity and the corresponding equivalence with the strongly monotonicity in the setting of Riemannian manifolds. Throughout the whole section, let $f: M \rightarrow(-\infty,+\infty]$ be a proper convex function. Recall from [13] that the subdifferential $\partial f: M \rightarrow 2^{T_{x} M}$ of $f$ is defined by

$$
\partial f(x):=\left\{u \in T_{x} M \mid\langle u, \dot{\gamma}(0)\rangle \leq f(y)-f(x), \forall y \in \mathrm{D}(f) \text { and } \gamma \in \Gamma_{x y}^{(f)}\right\},
$$

for each $x \in \mathrm{D}(f)$ (otherwise, $\partial f(x):=\emptyset$ ). Let $x \in \mathrm{D}(f)$ and $v \in T_{x} M$. Define the directional derivative at $x$ in the direction $v$ by

$$
f^{\prime}(x ; v):=\lim _{t \rightarrow 0^{+}} \frac{f\left(\exp _{x} t v\right)-f(x)}{t}
$$

Then we have the following assertions:

Proposition 6.1. Let $f: M \rightarrow(-\infty,+\infty)$ be convex and $x \in M$. Then the following assertions hold.

(i) $\partial f(x)=\left\{u \in T_{x} M \mid\langle u, v\rangle \leq f^{\prime}(x ; v)\right.$ for all $\left.v \in T_{x} M\right\}$.

(ii) $f^{\prime}(x, v)=\sup _{u \in \partial f(x)}\langle u, v\rangle$, for each $v \in T_{x} M$.

Proof. (i) has been proved in [35]. We next show that (ii) is true. Recall from [35, Proposition 3.5(iii)] that the support function of the subdifferential is the lower semicontinuous hull of the directional derivative $f^{\prime}(x ; \cdot)$ of $f$ at $x$; that is,

$$
\sup _{u \in \partial f(x)}\langle u, \cdot\rangle=\operatorname{cl} f^{\prime}(x ; \cdot) .
$$


Note that $\mathrm{D}(f)=M$. We have $\mathrm{D}\left(f^{\prime}(x ; \cdot)\right)=T_{x} M$. Recall also from [35, Proposition $3.5(\mathrm{i})]$ that $f^{\prime}(x ; \cdot)$ is convex. Thus it follows that

$$
\operatorname{cl} f^{\prime}(x ; \cdot)=f^{\prime}(x ; \cdot)
$$

Hence (ii) is seen to hold.

Definition 6.2. Let $\alpha>0$. The function $f$ is said to be $\alpha$-strongly convex if, for any $x, y \in \mathrm{D}(f)$ and any geodesic $\gamma \in \Gamma_{x y}^{(f)}$, the following inequality holds:

$$
f(\gamma(t)) \leq(1-t) f(x)+t f(y)-\frac{1}{2} \alpha t(1-t) l^{2}(\gamma) \quad \text { for each } 0<t<1
$$

The following theorem is an extension of Rockafellar's result to the setting of Riemannian manifolds.

Theorem 6.3. Let $f: M \rightarrow \mathbb{R}$ be a convex function and let $\alpha>0$. Then $f$ is $\alpha$-strongly convex if and only if $\partial f$ is $\alpha$-strongly monotone.

Proof. Let $x, y \in \mathrm{D}(f)$ and $\gamma \in \Gamma_{x y}^{(f)}$. Define the function $F_{\gamma}:[0,1] \rightarrow \mathbb{R}$ by

$$
F_{\gamma}(t):=f(\gamma(t)) \text { for each } t \in[0,1] .
$$

Then, for each $t \in[0,1]$, we have that

$$
F_{\gamma}^{\prime}(t,-1)=f^{\prime}(\gamma(t),-\dot{\gamma}(t)), \quad F_{\gamma}^{\prime}(t, 1)=f^{\prime}(\gamma(t), \dot{\gamma}(t))
$$

and

$$
\partial F_{\gamma}(t)=\left[-F_{\gamma}^{\prime}(t,-1), \quad F_{\gamma}^{\prime}(t, 1)\right]=\left[-f^{\prime}(\gamma(t),-\dot{\gamma}(t)), \quad f^{\prime}(\gamma(t), \dot{\gamma}(t))\right] .
$$


It follows that $\partial F_{\gamma}$ is $\alpha l^{2}(\gamma)$-strongly monotone if and only if

$$
\left(-f^{\prime}\left(\gamma\left(t_{2}\right),-\dot{\gamma}\left(t_{2}\right)\right)-f^{\prime}\left(\gamma\left(t_{1}\right), \dot{\gamma}\left(t_{1}\right)\right)\right)\left(t_{2}-t_{1}\right) \geq \alpha l^{2}(\gamma)\left(t_{2}-t_{1}\right)^{2}
$$

holds for any $0 \leq t_{1} \leq t_{2} \leq 1$. By Proposition 6.1 we have that

$$
-f^{\prime}\left(\gamma\left(t_{2}\right),-\dot{\gamma}\left(t_{2}\right)\right)-f^{\prime}\left(\gamma\left(t_{1}\right), \dot{\gamma}\left(t_{1}\right)\right)=\inf _{w_{2} \in \partial f\left(\gamma\left(t_{2}\right)\right)}\left\langle w_{2}, \gamma^{\prime}\left(t_{2}\right)\right\rangle-\sup _{w_{1} \in \partial f\left(\gamma\left(t_{1}\right)\right)}\left\langle w_{1}, \gamma^{\prime}\left(t_{1}\right) .\right.
$$

Combining (34) and (35), one sees that $\partial F_{\gamma}$ is $\alpha l^{2}(\gamma)$-strongly monotone if and only if

$$
\left(\left\langle w_{2}, \gamma^{\prime}\left(t_{2}\right)\right\rangle-\left\langle w_{1}, \gamma^{\prime}\left(t_{1}\right)\right\rangle\right)\left(t_{2}-t_{1}\right) \geq \alpha l^{2}(\gamma)\left(t_{2}-t_{1}\right)^{2}, \quad \forall w_{2} \in \partial f\left(\gamma\left(t_{2}\right)\right), \forall w_{1} \in \partial f\left(\gamma\left(t_{1}\right)\right)
$$

holds for any $0 \leq t_{1} \leq t_{2} \leq 1$. Hence, we get the following fact that for any $x, y \in \mathrm{D}(f)$ and any geodesic $\gamma \in \Gamma_{x y}^{(f)}, \partial F_{\gamma}$ is $\alpha l^{2}(\gamma)$-strongly monotone if and only if $\partial f$ is $\alpha$ strongly monotone. Furthermore, by definition, it's easy to verify that $f$ is $\alpha$-strongly convex if and only if, for any $x, y \in \mathrm{D}(f)$ and any geodesic $\gamma \in \Gamma_{x y}^{(f)}$, the function $F_{\gamma}$ is $\alpha l^{2}(\gamma)$-strongly convex on $[0,1]$. By [26, Proposition 6] (applied to the function $F_{\gamma}$ ), one sees that $F_{\gamma}$ is $\alpha l^{2}(\gamma)$-strongly convex if and only if $\partial F_{\gamma}$ is $\alpha l^{2}(\gamma)$-strongly monotone. Thus the conclusion of this theorem follows.

Below, we show that, for each fixed $y \in M$, the vector field $T: M \rightarrow T M$ defined by

$$
T(x):=-\exp _{x}^{-1} y, \text { for each } x \in M,
$$

is 1-strongly monotone. This fact has been previously proved in [36]. However, as an example of the application of Theorem 6.3, we give a different proof. 
Example 6.1. Let $M$ be a Hadamard manifold and let $y \in M$ be fixed. Consider the function $\phi: M \rightarrow \mathbb{R}$ defined by

$$
\phi(x)=\frac{1}{2} \mathrm{~d}^{2}(y, x), \quad \text { for each } x \in M
$$

Then by [29], $\phi$ is differential and its derivative is

$$
\phi^{\prime}(x)=\partial \phi(x):=-\exp _{x}^{-1} y \quad \text { for each } x \in M
$$

Hence we have that $T=\phi^{\prime}$. We will prove that $\phi$ is 1-strongly convex on $M$ and therefore, by Theorem 6.3, $\phi^{\prime}$ is 1-strongly monotone on $M$. For this aim, take $x, z \in M$ and $\gamma \in \Gamma_{x z}$. Then $\gamma(0)=x$ and $\gamma(1)=z$. Consider the geodesic triangle $\Delta(x y z)$ and the corresponding comparision one $\Delta(\bar{x} \bar{y} \bar{z})$ in $\mathbb{R}^{2}$ (cf. [37, p.24]) such that

$$
\mathrm{d}(x, y)=\|\bar{x}-\bar{y}\|, \quad \mathrm{d}(y, z)=\|\bar{y}-\bar{z}\| \quad \text { and } \quad \mathrm{d}(x, z)=\|\bar{x}-\bar{z}\| .
$$

Let $t \in[0,1]$ and let $\overline{\gamma(t)}:=(1-t) \bar{x}+t \bar{z}$ denote the point in $\mathbb{R}^{2}$ corresponding to $\gamma(t)$. Then, by [38, Lemma 4.3],

$$
\mathrm{d}^{2}(y, \gamma(t)) \leq\|\bar{y}-\overline{\gamma(t)}\|^{2}=\|\bar{y}-[(1-t) \bar{x}+t \bar{z}]\|^{2}
$$

Since

$$
\|\bar{y}-[(1-t) \bar{x}+t \bar{z}]\|^{2}=(1-t)\|\bar{x}-\bar{y}\|^{2}+t\|\bar{z}-\bar{y}\|^{2}-t(1-t)\|\bar{x}-\bar{z}\|^{2},
$$

It follows from (37) and (38) that

$$
\mathrm{d}^{2}(y, \gamma(t)) \leq(1-t) \mathrm{d}^{2}(y, x)+t \mathrm{~d}^{2}(y, z)-t(1-t) \mathrm{d}^{2}(x, z)
$$

which implies that $\phi$ is strongly convex with modulus $\alpha=1$. 
By Theorems 5.1 and 6.3, the following theorem is immediate.

Theorem 6.4. Let $f: M \rightarrow \mathbb{R}$ be $\alpha$-strongly convex. Suppose that $f$ is continuously differentiable and its gradient $\nabla f$ is L-Lipschitz continuous. Then, for any $x_{0} \in M$, the sequence $\left\{x_{n}\right\}$ defined by the algorithm

$$
x_{n+1}=\exp _{x_{n}}\left(-r \nabla f\left(x_{n}\right)\right)
$$

where $0<r<\frac{2 \alpha}{L^{2}}$, converges to a minimizer of $f$ in $M$.

\section{References}

[1] Browder, F. E., Petryshyn, W. V.: Construction of fixed points of nonlinear mappings in Hilbert spaces, J. Math. Anal. Appl. 73, 875-881 (1967)

[2] Brezis, H.: Opérateurs Maximaux Monotones et Semi-groupes de Contractions Dans Les Espaces de Hilbert. American Elsevier Publishing Co., Inc., New York (1973)

[3] Zeidler, E.: Nonlinear Functional Analysis and Applications. II/B. Nonlinear Monotone Operators. Springer Verlag, New York (1990)

[4] Cioranescu, I.: Geometry of Banach Spaces, Duality Mappings and Nonlinear Problems. Kluwer Academic Publishers, Dordrecht (1990)

[5] Da Cruz Neto, J. X., Ferreira, O. P. , Lucambio Pérez, L. R.: Monotone point-to-set vector fields, Balkan Journal of Geometry and its Applications. 5, 69-79 (2000) 
[6] Ferreira, O. P., Oliveira, P. R.: Proximal point algorithm on Riemannian manifolds. Optim. 51, 257-270 (2002)

[7] Ferreira, O. P., Lucambio Pérez, L. R., Nemeth, S. Z.: Singularities of monotone vector fields and an extragradient-type algorithm, J. Global Optim. 31, 133-151 $(2005)$

[8] Rapcsk, T.: Smooth Nonlinear Optimization in $\mathbb{R}^{n}$, Nonconvex Optimization and its Applications, 19, Kluwer Academic Publisher, Dordrecht (1997)

[9] Martín-Márquez, V.: Nonexpansive mappings and monotone vector fields in Hadamard manifold, Communications in Applied Analysis 13, 633-646, (2009)

[10] Smith, S. T.: Optimization techniques on Riemannian manifolds, Fields Institute Communications 3, American Mathematical Society, Providence, R. I., 113-146 (1994)

[11] Sturm, K. T.: Probability Measures on Metric Spaces of Nonpositive Curvature. Heat kernels and Analysis on Manifolds, Graphs, and Metric Spaces. (Paris, 2002) 357-390, Contemp. Math., 338, Amer. Math. Soc., Providence, RI (2003)

[12] Iwamiya, T., Okochi, H.: Monotonicity, resolvents and Yosida approximations of operators on Hilbert manifolds, Nonlinear Anal. 54, 205-214 (2003) 
[13] Udriste, C.: Convex Functions and Optimization Methods on Riemannian Manifolds. Mathematics and Its Applications, 297, Kluwer Academic Publisher, Dordrecht (1994)

[14] Li, S. L., Li, C, Liou, Y. C., Yao, J. C.: Existence of solutions for variational inequalities on Riemannian manifolds, Nonliear Anal. 71(11), 5695-5706 (2009)

[15] Ledyaev, Y. S., Zhu, Q. J.: Nonsmooth analysis on smooth manifolds, Trans. Amer. Math. Soc. 359, 3687-3732 (2007)

[16] Dedieu, J. P. et al.: Newton's method on Riemannian manifolds: covariant alpha theory, IMA J. Numer. Anal. 23, 395-419 (2003)

[17] Ferreira, O. P., Svaiter, B. F.: Kantorovich's theorem on Newton's method in Riemannian manifolds, J. Complexity 18, 304-329 (2002)

[18] Li, C., Wang, J. H.: Convergence of the Newton method and uniqueness of zeros of vector fields on Riemannian manifolds, Sci. China Ser. A. 48, 1465-1478 (2005)

[19] Li, C., Wang, J. H.: Newton's method on Riemannian manifolds: Smale's point estimate theory under the $\gamma$-condition, IMA J. Numer. Anal. 26, 228-251 (2006)

[20] Li, C., Wang, J. H.: Newton's method for sections on Riemannian manifolds: Generalized Covariant $\alpha$-Theory, J. Complexity 24, 423-451 (2008)

[21] Wang, J. H., Li, C.: Uniqueness of the singular points of vector fields on Riemannian manifolds under the $\gamma$-condition, J. Complexity 22, 533-548 (2006) 
[22] Azagra, D., Ferrera, J., López-Mesas, F.: Nonsmooth analysis and Hamilton-Jacobi equations on Riemannian manifolds. J. Funct. Anal. 220, 304-361 (2005)

[23] Li, C., López, G., Martín-Márquez, V.: Monotone vector fields and the proximal point algorithm on Hadamard manifols, J. London Math. Soc. 79(3), 663-683 (2009)

[24] Németh, S. Z.: Monotone vector fields, Publicationes Mathematicae Debrecen. 54(34), 437-449 (1999)

[25] Németh, S. Z.: Geodesic monotone vector fields, Lobachevskii Journal of Mathematics. 5, 13-28 (1999)

[26] Rockafellar, R. T.: Monotone operators and the proximal point algorithm, SIAM J. Control Optim. 14, 877-898 (1976)

[27] DoCarmo, M. P.: Riemannian Geometry. Birkhauser, Boston (1992)

[28] Sakai, T.: Riemannian Geometry. Translations of Mathematical Monographs 149, American Mathematical Society, Providence, RI (1996)

[29] Walter, R.: On the metric projection onto convex sets in Riemannian spaces, Arch. Math. 25, 91-98 (1974)

[30] Cheeger, J., D. Gromoll, D.: On the structure of complete manifolds of nonnegative curvature, Ann. of Math. 96(2), 413-443 (1972)

[31] Chidume, C. E.: Iterative approximation of fixed points of Lipschitzian strictly pseudo-contractive mappings, Proc. Amer. Math. Soc. 99(2), 283-288 (1987) 
[32] Chidume, C. E.: Iterative approximation of the solution of a monotone operator equation in certain Banach spaces, International Centre for theorical Phisics IC $/ 88 / 22(1988)$

[33] Kamimura, S., Takahashi, W.: Approximating solutions of maximal monotone operators in Hilbert spaces, J. Approx. Theory. 13, 226-240 (2000)

[34] Polyak, B. T.: Existence theorems and convergence of minimizing sequences in extremun problems with restrictions, Dokl. Akad. Nauk. USSR. 166, 287-290 (1966)

[35] Wang, J. H., Li, C., Mordukhovich, B. S., Yao, J. C.: Weak sharp minima on Riemannian manifolds, submitted

[36] Da Cruz Neto, J. X., Ferreira, O. P. , Lucambio Pérez, L. R.: Contributions to the study of monotone vector fields, Acta Math. Hungar. 94 (4), 307-320 (2002)

[37] Bridson, M., Haefliger, A.: Metric Spaces of Non-positive Curvature. SpringerVerlag, Berlin, Heidelberg, New York (1999)

[38] Li, C., López, G., Martín-Márquez, V.: Iterative algorithms for nonexpansive mappings in Hadamard manifolds. Taiwanese J. Math. to appear in 2010 